\title{
Chimeric cDNA Sequences from Citrus tristeza virus Confer RNA Silencing- Mediated Resistance in Transgenic Nicotiana benthamiana Plants
}

\author{
Gourgopal Roy, Mysore R. Sudarshana, Diane E. Ullman, \\ Shou-Wei Ding, Abhaya M. Dandekar, and Bryce W. Falk
}

\begin{abstract}
First, second, and sixth authors: Department of Plant Pathology, third author: Department of Entomology, and fifth author: Department of Plant Science, University of California, Davis; and fourth author: Department of Plant Pathology, University of California, Riverside. Current address of G. Roy: Center for Molecular Biotechnology, Fraunhofer USA, Newark, DE 19711. Accepted for publication 31 March 2006.
\end{abstract}

\section{ABSTRACT}

Roy, G., Sudarshana, M. R., Ullman, D. E., Ding, S.-W., Dandekar, A. M., and Falk, B. W. 2006. Chimeric cDNA sequences from Citrus tristeza virus confer RNA silencing-mediated resistance in transgenic Nicotiana benthamiana plants. Phytopathology 96:819-827.

RNA silencing has been shown to be an important mechanism for conferring resistance in transgenic, virus-resistant plants. We used this approach to evaluate resistance in Nicotiana benthamiana plants transformed with chimeric coding and noncoding sequences from Citrus tristeza virus (CTV). Several independent transgenic plant lines were generated, using two constructs (pCTV1 and pCTV2) designed to produce self-complementary transcripts. The pCTV1 contained cDNA sequences from the CTV capsid protein (CP), p20, and 3' untranslated region (UTR); and pCTV2 contained $\mathrm{CP}, \mathrm{p} 23$, and $3^{\prime}$ UTR sequences.
Heterologous recombinant Potato virus $X$ (PVX) containing either homologous or heterologous CTV sequences was used to challenge plants and resistance was evaluated phenotypically and validated with reversetranscriptase polymerase chain reaction and northern hybridization analysis. Transgenic plants (T1 generation) for each construct showed resistance to recombinant PVX constructs used for challenge experiments when PVX contained p20 or UTR (for CTV1 plants), or p23 or UTR (for CTV2 plants). However, no resistance was seen when plants were challenged with PVX containing the CTV CP. T2 generation plants also showed resistance even when challenged with PVX containing the cognate CTV sequences obtained from heterologous CTV isolates. The presence of transgene-specific small interfering RNAs in the resistant CTV1 and CTV2 plants indicated that resistance was mediated by posttranscriptional gene silencing.
RNA silencing refers to a conserved eukaryotic pathway of posttranscriptional suppression of gene expression by sequencespecific degradation of specific messanger (m)RNA. This first was observed in plants and termed posttranscriptional gene silencing (PTGS) (29). RNA silencing can be induced by structured single-stranded RNA or double-stranded RNA derived from a replicating virus, transgene, or aberrant RNAs that are processed into small interfering RNAs (siRNA) by a Dicer-like enzyme (4,9, 16,28,39). These siRNAs guide sequence-specific target mRNA degradation by the RNA-induced silencing complex (RISC) (17, 30). In 1992, RNA silencing was shown to confer a high level of resistance to Tobacco etch virus, a member of the genus Potyvirus, in transgenic plants $(22,24)$. Since then, RNA silencing also has been shown to confer high levels resistance to several other RNA viruses in transgenic plants $(33,36,37)$. Various aspects of this have been reviewed recently $(23,26)$.

Tristeza, one of the most destructive citrus diseases worldwide $(2,31)$, is caused by Citrus tristeza virus (CTV), a member of the genus Closterovirus in the family Closteroviridae. The CTV genome is a plus-sense, single-stranded RNA of $\approx 19.5 \mathrm{~kb}$ and has 12 open reading frames (ORFs) encoding at least 17 different proteins $(19,20)$. Three of the CTV-encoded proteins, p23, p20, and capsid protein (CP), are suppressors of PTGS activity (25). The p23 and CP function intracellularly and intercellularly, respectively, whereas p20 acts both ways (25). CTV can be present in trees as mixed and complex infections; and many different strains

Corresponding author: B. W. Falk; E-mail address: bwfalk@ucdavis.edu

DOI: 10.1094/PHYTO-96-0819

(C) 2006 The American Phytopathological Society and sequence variants have been reported. Thus, strategies for CTV control must consider these properties if effective control is to be achieved.

Several traditional practices, such as eradication of CTV-infected trees, planting "certified" CTV-free material, and crossprotection using mild strains (6), can minimize CTV-induced disease losses, but none are effective in completely controlling CTV. Recent attempts, including studies with markers linked to the CTV resistance gene from Poncirus trifoliata (3), quantitative trait loci (QTL) analysis (1), and biotechnological approaches such as genetically engineered citrus plants containing specific CTV genes (e.g., coat protein gene), have been used to attempt to develop CTV resistance; however, so far, none have been reported as providing adequate protection $(7,12,15)$.

In the present study, we used chimeric self-complementary CTV constructs (pCTV1 and pCTV2) as transgenes in attempts to evaluate PTGS as a possible strategy to confer resistance against Potato virus $X$ (PVX) containing homologous CTV sequences, and sequences from distinct CTV sequence variants. Each silencing construct contained highly conserved CTV sequences targeting two of the three CTV-encoded silencing suppressors. We transformed Nicotiana benthamiana plants and showed that both local and systemic virus infection can be impaired after challenging with recombinant PVX containing homologous or heterologous CTV sequences.

\section{MATERIALS AND METHODS}

CTV isolates. We used a naturally occurring California CTV isolate, CA-65 (21), for making the chimeric CTV1 and CTV2 constructs. For the heterologous PVX challenge experiments, we collected geographically different CTV isolates from the U.S. 
Department of Agriculture Beltsville collection of exotic CTV isolates (Beltsville, MD).

Reverse-transcriptase polymerase chain reaction and cloning of CTV-specific sequences. Total RNA was isolated from CTV-infected citrus using TRI reagent (MRI Inc., Cincinnati, $\mathrm{OH})$. Primers for amplification of the genome segments, including restriction sites to facilitate cloning, are shown in Table 1. Specific sequences from conserved regions of CP, p20, p23, and $3^{\prime}$ untranslated region (UTR) nucleotide sequences were amplified from total RNA by two-step reverse-transcriptase polymerase chain reaction (RT-PCR) using AMV reverse transcriptase ( 1 cycle of $42^{\circ} \mathrm{C}$ for $1 \mathrm{~h}$ ) and $P f u$ DNA polymerase ( 1 cycle of $95^{\circ} \mathrm{C}$ for $2 \mathrm{~min} ; 1$ cycle of $95^{\circ} \mathrm{C}$ for $30 \mathrm{~s}, 45^{\circ} \mathrm{C}$ for $30 \mathrm{~s}$, and $72^{\circ} \mathrm{C}$ for $1 \mathrm{~min}$; and 29 cycles of $94^{\circ} \mathrm{C}$ for $30 \mathrm{~s}, 50^{\circ} \mathrm{C}$ for $30 \mathrm{~s}$, and $72^{\circ} \mathrm{C}$ for $1 \mathrm{~min}$; followed by 1 cycle of $72^{\circ} \mathrm{C}$ for $10 \mathrm{~min}$ ). Purified $\mathrm{CP}$

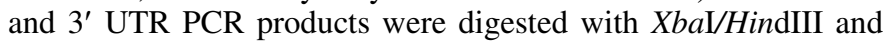
SalI/BamHI respectively, whereas the p20 and p23 fragments were digested with HindIII/SalI. Fragments with compatible ends were gel purified and ligated into XbaI- and BamHI-digested pBluescript II SK (Stratagene, La Jolla, CA) in a single ligation reaction. The $\mathrm{CP}, \mathrm{p} 20$, and 3' UTR sequences gave pGS1, and CP, p23, and 3' UTR gave pGS2 (Fig. 1).

The chimeric insert CP-p20-UTR from pGS1 was digested independently with XhoI and EcoRI (for sense orientation) and with $X b a I$ and BamHI (for antisense orientation). These fragments, plus XhoI- and XbaI-digested pRNA69 (13) and the EcoRIand BamHI-digested 632-bp intron fragment of pRNA69, were mixed and ligated. This generated clone pGSG8. Similarly, clone pGSG29 was generated by inserting the CP-p23-UTR fragments (from pGS2) in both sense and antisense orientation into pRNA69 as described above (Fig. 1).

Generation of binary vector and transgenic plants. Each of these constructs, pGSG8 and pGSG29, were digested with NotI and the desired fragment ligated independently into the Not I digested binary vector (pDU97.1005) (a modified version of pCGN1547; S. Uratsu and A. M. Dandekar, unpublished data) (27). The binary silencing constructs made with pGSG8 and pGSG29 were designated as pCTV1 and pCTV2, respectively (Fig. 1), and their corresponding transgenic lines were described as CTV1 and CTV2, respectively.

Primary transgenic plants (T0) were analyzed by PCR using both internal (CTV sequence specific; primers 5CPXXh and UTR3BE) and external (promoter and intron specific; primers RN69-1307 and RN69-1436, RN69-1958 and RN69-2057) primers. Plants were allowed to self-pollinate and were grown for seed in the greenhouse. Transgenic seeds were selected on kanamycin (kanamycin sulfate at 100 or $150 \mathrm{mg} /$ liter) containing media (half-strength Murashige and Skoog Basal Salts with minimal organics; Sigma-Aldrich, Inc., St. Louis). Surviving plants for each transgenic line were used for screening experiments.

PVX constructs, in vitro transcription, and plant inoculation. The PVX clone pPC2S (a generous gift from D. C. Baulcombe, The Sainsbury Laboratory, Norwich, UK) was used to generate different constructs (PVX-CP, PVX-p20, PVX-p23, PVX-UTR, and PVX-GFP [Fig. 2A] and PVX-CPUTR, PVX-CPp20, and PVX-p20UTR [Fig. 2B]). Specific CTV cDNA sequences were ligated into $\mathrm{pPC} 2 \mathrm{~S}$ in the sense orientation. Clones were confirmed by nucleotide sequence analysis. The $\mathrm{T} 7 \mathrm{mMessage}$ mMachine kit (Ambion, Foster City, CA) was used for all in vitro transcription reactions. Infectious RNAs were generated from 1 to $2 \mu \mathrm{g}$ of SpeI-linearized plasmid DNA. Inoculations were done by mixing $2 \mu \mathrm{l}$ of transcript with $100 \mu \mathrm{l}$ of FES buffer (1\% sodium pyrophosphate, $1 \%$ bentonite, $1 \%$ celite in $0.1 \mathrm{M}$ glycine and $0.06 \mathrm{M}$ dibasic potassium phosphate) and at least two nontransgenic $N$. benthamiana plants were inoculated for each PVX construct. The infected inoculated leaves were harvested at 7 days postinoculation and confirmed for the presence of desired insert sequence by RT-PCR using PVX-specific primers (Table 1). Leaves were frozen at $-80^{\circ} \mathrm{C}$ and used as the source of inoculum for all the experiments.

Eighteen plants per transgenic line were used to evaluate resistance. Inocula were prepared by grinding virus-infected leaf material in $0.03 \mathrm{M}$ potassium phosphate buffer, $\mathrm{pH} 7.0$, containing $0.1 \%$ sodium sulfite. Plants were inoculated at the four- to six-leaf stage and monitored daily for symptoms. Data were recorded at 2, $6,7,10,15,16$, and 20 days postinoculation. In addition to symptoms, plants were screened for virus infection by using RT-PCR. At least 10 plants per line and per construct were analyzed by RTPCR using PVX-specific primers (PVX1 and PVX2, PVXCPF, and PVXCPR) (Table 1).

RNA extraction and northern hybridization analysis. Total RNA was isolated from 0.5 to $1 \mathrm{~g}$ of leaf tissue using Trizol (Invitrogen, Carlsbad, CA). Low molecular weight (LMW) RNA was isolated by precipitating RNA with $10 \%$ PEG-8000 and $0.5 \mathrm{M}$ $\mathrm{NaCl}$, followed by ethanol precipitation. The LMW RNA was quantified by UV absorbance and by nondenaturing agarose (2\%) gel electrophoresis. LMW RNA $(\approx 5$ to $10 \mu \mathrm{g})$ was used for denaturing polyacrylamide gel (20\% polyacrylamide [19:1], and $8 \mathrm{M}$ urea) electrophoresis. The gels were stained in ethidium bromide and RNAs were electroblotted onto Hybond-NX (Amersham Pharmacia, Piscataway, NJ). The membrane was dried at $80^{\circ} \mathrm{C}$ in an oven for $30 \mathrm{~min}$ and the RNA was fixed by UV crosslinking (Stratagene UV Crosslinker). For probes CP-p20UTR and CP-p23-UTR, we used plasmids pGS1 and pGS2

TABLE 1. Oligonucleotides used in polymerase chain reaction for making transgene constructs and detection of recombinant Potato virus $X$ (PVX)

\begin{tabular}{|c|c|c|}
\hline Primers & Nucleotide sequence $\left(5^{\prime}-3^{\prime}\right)^{\mathrm{a}}$ & Specificity and orientation ${ }^{\mathrm{b}}$ \\
\hline $5 \mathrm{CPXXh}$ & ACTTTCTAGACTCGAGCTGGGGTAGGACTATCGA & CTV-CP sense \\
\hline 3CPHIII & ACATAAGCTTTCAACGTGTGTTGAATTTCC & CTV-CP antisense \\
\hline $5 \mathrm{P} 20 \mathrm{HIII}$ & TACAAAGCTTATGCGAGCTTACTTTAGTGT & CTV-p20 sense \\
\hline $3 \mathrm{P} 20 \mathrm{~S}$ & TACAGTCGACCTACACGCATGAAGGAGAA & CTV-p20 antisense \\
\hline UTR5S & TAATGTCGACTTGAAGTGGACGGAATAAG & CTV-UTR sense \\
\hline UTR3BE & TAATGGATCCGAATTCTGGACCTATGTTGGCC & CTV-UTR antisense \\
\hline $5 \mathrm{P} 23 \mathrm{HIII}$ & ATCTAAGCTTGATTATACTAGCGGACAAAC & CTV-p23 sense \\
\hline $3 \mathrm{P} 23 \mathrm{~S}$ & ATCTETCGACGATGAAGTGGTGTTCACG & CTV-p23 antisense \\
\hline RN69-1307 & TTCGCAAGACCCTTCCTGTAT & $35 \mathrm{~S}$ promoter sense \\
\hline RN69-1436 & CTGTTTGAACATATAAAAGAAATAAAAACT & Spacer antisense \\
\hline RN69-1958 & TGTAGTGGGCACACTTTCCG & Spacer sense \\
\hline RN69-2057 & AAATATCATGCGATCATAGGCG & Ocs promoter antisense \\
\hline PVX1 & CCATTGCCGATCTCAAGCCACTC & PVX-CP sense \\
\hline PVX2 & GTAGTTGAGGTAGTTGACCCTATG & PVX-CP antisense \\
\hline PVXCPF & TTGGAAGGACATGAAGGTGC & PVX-CP sense \\
\hline PVXCPR & TCAGCGGTTGTTGTTCCAGT & PVX-CP antisense \\
\hline
\end{tabular}

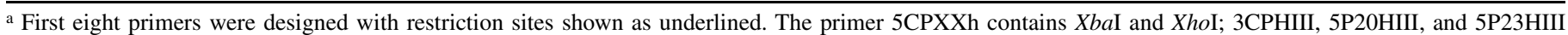
contain HindIII; 3P20S, UTR5S, and 3P23S contain SalI; and UTR3BE contains BamHI and EcoRI.

b Indicates polarity and Citrus tristeza virus (CTV) region targeted. 
that were linearized with $\mathrm{XbaI}$, and radiolabeled transcripts were generated by in vitro transcription using T7 RNA polymerase (Ambion T7 Megascript Kit) and ${ }^{32}$ P-UTP (Perkin Elmer, Wellesley, MA). A CTV CP-specific clone (32) was used for detection of CP-specific siRNAs. For p20- and p23-specific probes, plasmids pGS1 and pGS2, respectively, were digested with HindIII-SalI and each desired fragment was cloned into HindIII-SalI-digested pBluescript SK and linearized with HindIII followed by in vitro transcription from T7 promoter. For the UTR-specific probe, plasmid pGS1 was digested with SacI-PstI to release the chimeric insert (CP-p20-UTR) from pBluescript SK and religated into SacI-PstI-digested pGEM-T Easy vector (Promega Corp., Madison, WI). This was done to avoid a second SalI site in the plasmid pGS1. It then was linearized with SalI and the probe was radiolabeled using $\mathrm{T} 7$ promoter-mediated in vitro transcription and ${ }^{32} \mathrm{P}$-UTP. Both prehybridization and hybridization were done overnight at $40^{\circ} \mathrm{C}$ followed by washing at $50^{\circ} \mathrm{C}$. Blots were exposed to X-ray film (Kodak) with intensifying screens at $-80^{\circ} \mathrm{C}$.

\section{RESULTS}

Transgenic $N$. benthamiana plants expressing the chimeric pCTV1/pCTV2 constructs are resistant to the individual CTV sequences. Five primary transgenic lines (T0) for CTV1 (001,
003, 009, 011, and 015) and CTV2 (002, 007, 013, 018, and 041) were generated by Agrobacterium-mediated transformation (Fig. 1). Plants from each of these lines were analyzed by PCR to confirm transgene composition. In each case, the internal (CTV sequence-specific, 5CPXXh and UTR3BE) and external (vectorspecific, RN69-1307 and RN69-1436, RN69-1958 and RN692057) pairs of primers (Table 1) were used to detect the presence of the integrated sequences (data not shown). The primary transformants were self-pollinated to obtain seed, and T1 progeny (second generation) plants were selected for kanamycin resistance.

To evaluate resistance, we inoculated nontransgenic and T1 transgenic plants with PVX containing homologous CTV sequences (Fig. 2) and monitored symptom phenotypes. Initial screening of five T1 CTV1 lines (pCTV1, CP-p20-UTR) showed variable resistance against PVX-p20 inoculation. Lines 009 and 015 showed good resistance $(>50 \%)$, whereas lines 001,003 , and 011 showed fewer resistant plants (6.25 to $27.7 \%$ ) (Table 2$)$. As observed with T1 CTV1 lines, transgenic T1 CTV2 lines (pCTV2, CP-p23UTR) also exhibited resistance. All five CTV2 lines 002, 007, 013,018 , and 041 showed good resistance $(>50 \%)$ when inoculated with PVX containing the homologous p23 sequence (Table 2). Among these lines, 002 and 041 showed a higher percentage of resistant plants. Interestingly, both CTV1 and CTV2 transgenic lines showing good resistance also had high kanamycin segre-
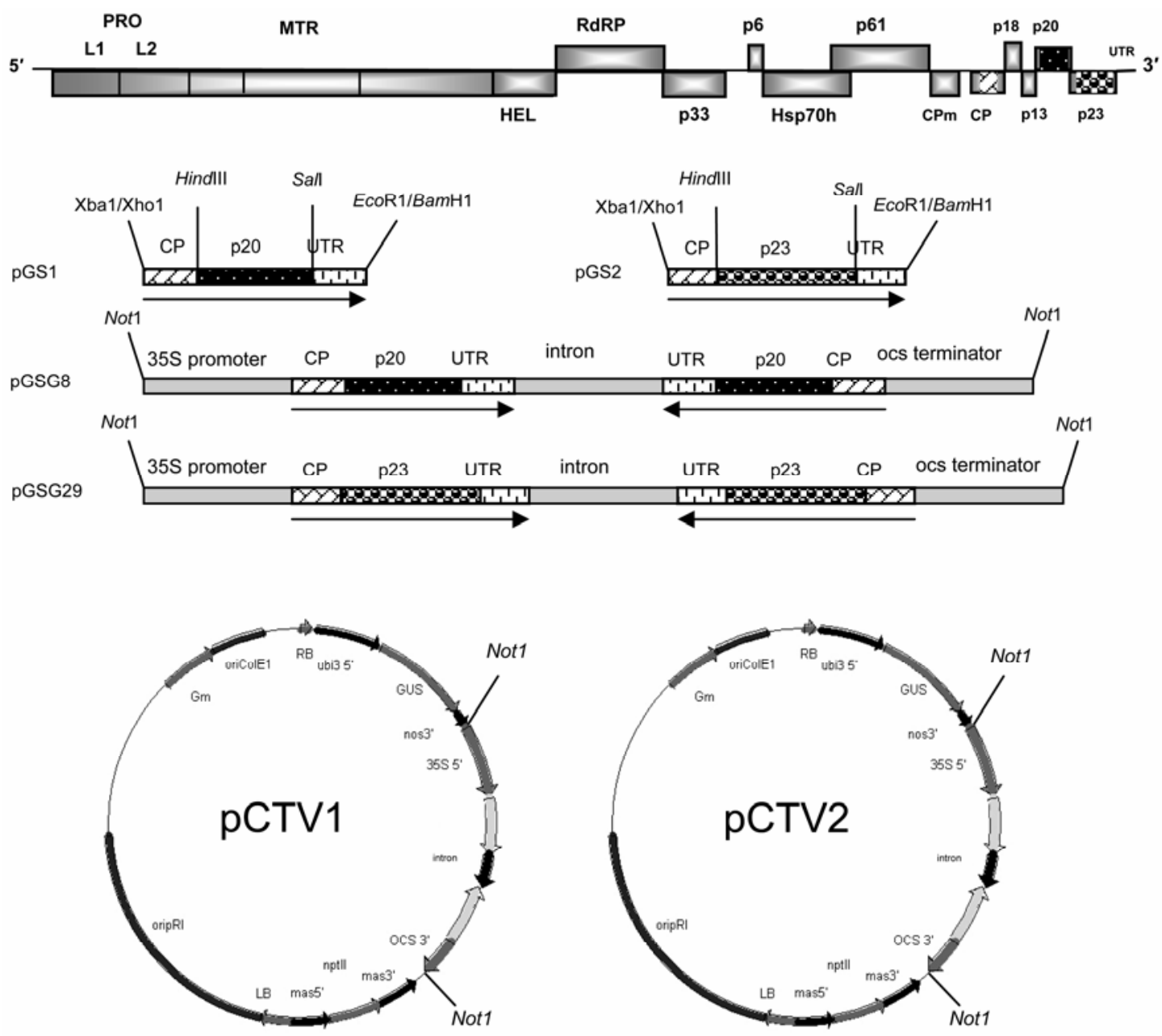

Fig. 1. Construction of the plasmids pGS1, pGS2, pGSG8, pGSG29, pCTV1, and pCTV2 used for plant transformation. The full-length Citrus tristeza virus (CTV) genomic map is shown at the top. Boxes indicate specific genes as indicated. The capsid protein (CP), p20, p23, and untranslated regions used for subsequent work are shown. The pGS1 and pGS2 are intermediate, chimeric plasmids. The pGSG8 contains chimeric insert from pGS1 in sense and antisense orientation separated by an intron, and includes a 35S promoter and an ocs terminator at its $5^{\prime}$ and $3^{\prime}$ end, respectively. The pGSG29 contains the chimeric insert from pGS2 in the same way as described for pGSG8. The binary constructs pCTV1 and pCTV2 are derived from pGSG8 and pGSG29, respectively, and contain all the necessary genes as indicated, including $\beta$-glucuronidase (GUS) and neomycin phosphotransferase (nptII). 
gation ratios, indicating multiple inserts (CTV1 lines 009 and 015 had ratios of 200:0 and 17:1, respectively; CTV2 lines 002 and 041 had ratios of 70:1 and 200:0, respectively). The resistant plants did not show any visible virus symptoms on either inoculated or upper, noninoculated leaves. As controls, all plants of the CTV1 and CTV2 lines also were inoculated with PVX containing CTV sequences not present in the transgene (PVX-p23 or PVXp20 for CTV1 or CTV2, respectively). All control plants developed mosaic symptoms typical of PVX infection by 7 days postinoculation, as did nontransgenic controls.
The phenotypic results were confirmed by RT-PCR analysis. Total RNA was extracted from each of 10 (randomly selected) transgenic plants of T1 CTV1 lines 009 and 015 that were inoculated with homologous PVX-p20, and RT-PCR was performed using PVX-specific primers (Table 1) designed to flank the recombinant insert (homologous p20 sequence). There was no amplification of target sequences from the resistant (nonsymptomatic) plants. However, amplification of the expected $\approx 690$-nucleotide (nt) products was obtained from all nontransgenic and symptomatic transgenic plants (Fig. 3). Similarly, for T1 CTV2 plants,
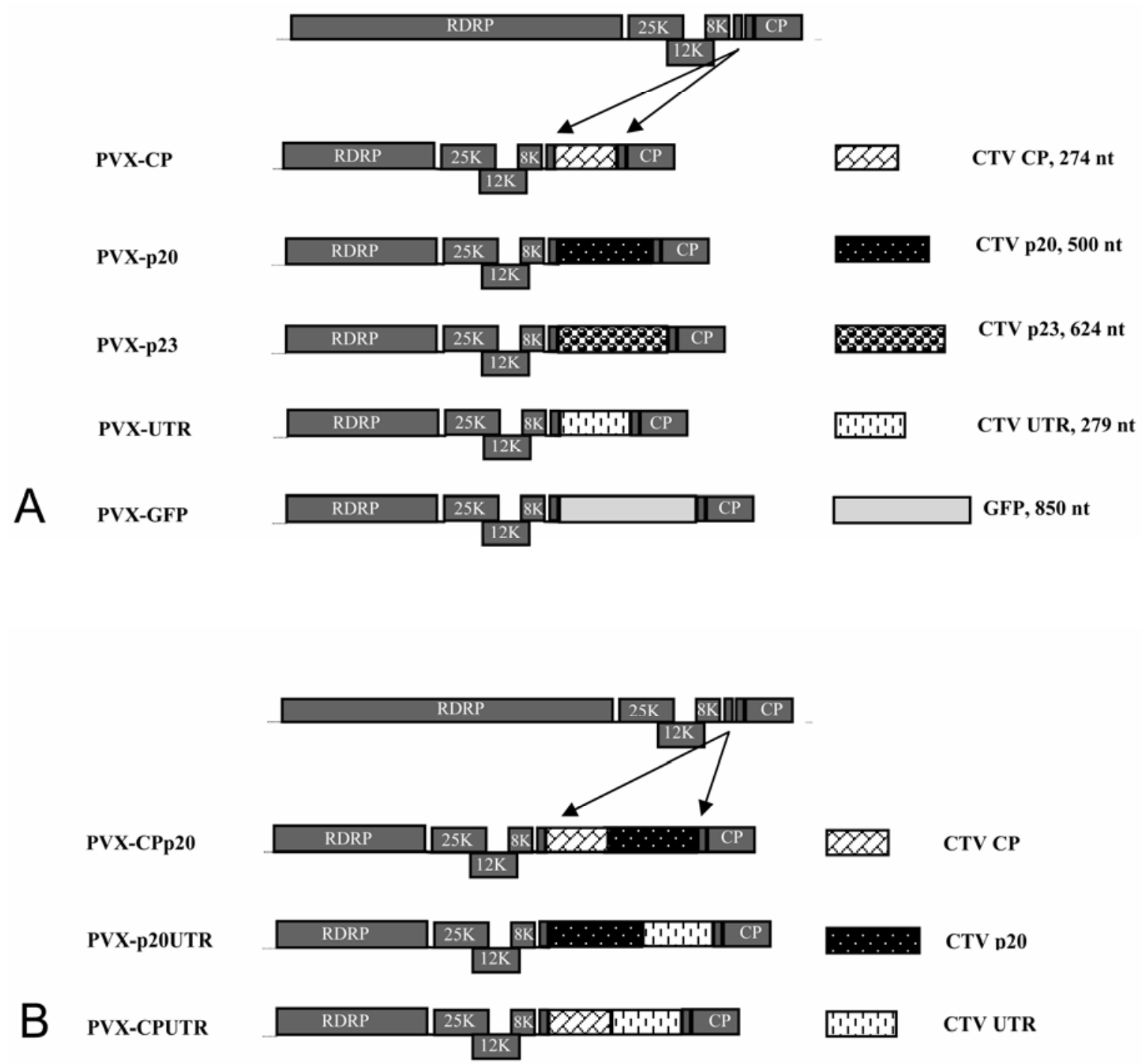

Fig. 2. Generation of recombinant Potato virus X (PVX) constructs. A, Construction maps for PVX-capsid protein (CP), PVX-p20, PVX-p23, PVX-untranslated region (UTR), and PVX-GFP, and B, for PVX-CPp20, PVX-p20UTR, and PVX-CPUTR fusions. Specific Citrus tristeza virus (CTV) regions CP, p20, UTR, and p23 are indicated at right and the numbers corresponding to the region indicate size of the fragments which were subcloned into PVX at the site between duplicated coat protein promoters (shown as gray boxes). Names indicating the specific PVX constructs are shown at left. Transcripts were generated in vitro and used for plant inoculation.

TABLE 2. Resistance screening of T1 transgenic Nicotiana benthamiana plants against Potato virus $X$ (PVX) containing homologous Citrus tristeza virus (CTV) p20 and p23 sequences ${ }^{\text {a }}$

\begin{tabular}{|c|c|c|c|c|c|c|c|c|c|c|c|}
\hline \multirow[b]{2}{*}{ Inoculum $^{\mathrm{b}}$} & \multirow[b]{2}{*}{ Nontransgenic } & \multicolumn{5}{|c|}{ Transgenic T1 CTV1 lines } & \multicolumn{5}{|c|}{ Transgenic T1 CTV2 lines } \\
\hline & & 001 & 003 & 009 & 011 & 015 & 002 & 007 & 013 & 018 & 041 \\
\hline PVX & $0 / 18$ & $0 / 18$ & $0 / 18$ & $0 / 18$ & $0 / 18$ & $0 / 18$ & $0 / 18$ & $0 / 18$ & $0 / 18$ & $0 / 18$ & $0 / 18$ \\
\hline PVX-p20 & $0 / 18$ & $3 / 18$ & $5 / 18$ & $10 / 18$ & $1 / 16$ & $13 / 18$ & $0 / 18$ & $0 / 16$ & $0 / 16$ & $0 / 18$ & $0 / 18$ \\
\hline PVX-GFP & $0 / 18$ & NT & NT & $0 / 18$ & NT & $0 / 18$ & $0 / 18$ & NT & NT & NT & $0 / 18$ \\
\hline
\end{tabular}

a Number of nonsymptomatic plants at 20 days postinoculation/total number of inoculated plants; NT = not tested.

${ }^{\mathrm{b}}$ Figure 2 provides details of constructs used to generate the inocula. 
RT-PCR analysis for PVX-p23-inoculated nontransgenic plants produced an amplicon of $\approx 778 \mathrm{nt}$, but no products were obtained from the transgenic resistant plants (data not shown). These results were consistent with the phenotypic observations and confirmed that either PVX-p20 or PVX-p23 did not establish infection on the resistant (nonsymptomatic) transgenic CTV1 and CTV2 plants, respectively.

Transgenic $N$. benthamiana plants also are resistant to heterologous CTV sequences obtained from geographically diverse isolates. Because CTV is a worldwide problem and various CTV isolates exhibit nucleotide sequence and biological diversity, effective resistance strategies must consider this diversity. Therefore, we examined our CTV1 and CTV2 plants for resistance to recombinant PVX-containing sequences from some representative diverse CTV isolates. We choose CTV isolates FLT30 (from Florida), IND-B227 (India), and ISR-VT (Israel) that were genetically different $(11,10$, and $10 \%$, respectively, in nucleotides) from the California isolate CA-65 (used to construct the transgene), and their corresponding p20 fragments were cloned into PVX. Similarly, p23 fragments from isolates CA-SY568 (California), FL-T30 (Florida), and IND-B227 (India) were

$\begin{array}{llllllllllllllll}\mathrm{M} & 1 & 2 & 3 & 4 & 5 & 6 & 7 & 8 & 9 & 10 & 11 & 12 & 13 & 14 & \mathrm{M}\end{array}$

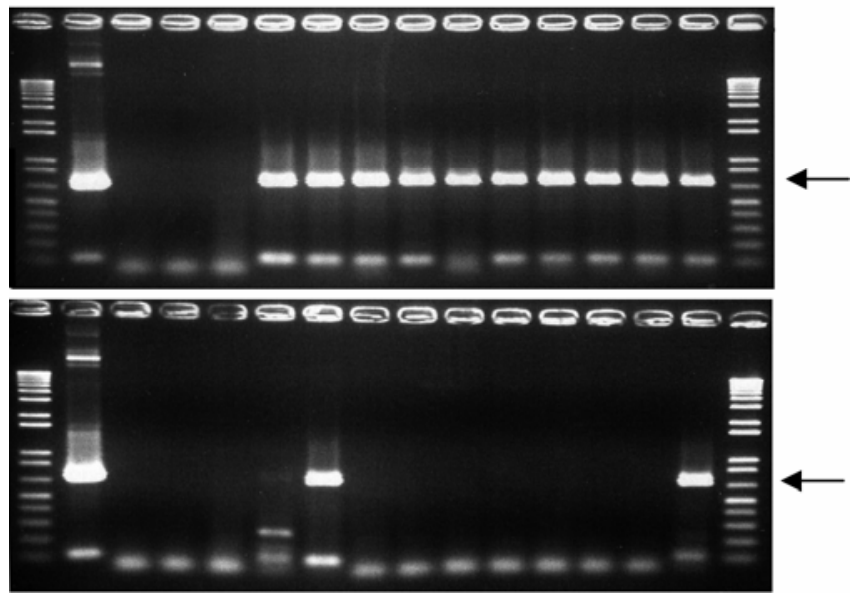

Fig. 3. Reverse-transcriptase polymerase chain reaction (RT-PCR) analysis for the presence of Potato virus $X$ (PVX)-p20 RNA in nontransgenic (top) and transgenic (bottom) Nicotiana benthamiana T1 Citrus tristeza virus (CTV)1 line 009 plants. M, 1-kb plus marker (Invitrogen); lane 1, PVX-p20 plasmid as positive control; lanes 2 and 3, uninoculated nontransgenic and transgenic control plants; lane 4, RT-PCR cocktail without template; lanes 5 through 14, 10 individual plants at 20 days postinoculation with PVX-p20. Arrows at left show position of the fragment corresponding to PVX-p20. No band indicates no PVX-p20. cloned into PVX. Each of these fragments differed from the corresponding transgene $\mathrm{p} 23$ sequence by $\approx 10 \%$. When plants were inoculated using the PVX-p20 constructs, both lines of the CTV1 plants showed resistance to the PVX constructs containing the homologous (CA-65) and heterologous p20 sequences (Table 3; Fig. 4A). In contrast, the nontransgenic control plants and CTV2 plants were susceptible to PVX-p20 (Table 3; Fig. 4A). Inoculation using the PVX-p23 constructs showed that the T2 CTV2 plants exhibited resistance to PVX constructs containing the homologous (CA-65) and heterologous p23 sequences, whereas nontransgenic control plants and CTV1 plants were susceptible (Table 3; Fig. 4B). We confirmed the resistance by RT-PCR (Fig. 5) and saw a perfect correlation between RT-PCR and the results assessed by phenotype.

Both CTV1 and CTV2 plants are susceptible to PVX-CP but not to PVX-UTR and PVX-CPUTR inoculations. Because the resistance seen above was evaluated only for PVX-p20 (CTV1) and PVX-p23 (CTV2), we were interested to assess whether other transgene components (PVX-CP and PVX-UTR) also showed resistance to viruses containing those transgene components. The CP and UTR sequences were those from the CTV isolate used to construct the chimeric transgenes in the CTV1 and CTV2 plants. T1 transgenic plants (both CTV1 and CTV2) showed good resistance when inoculated with PVX-UTR (Table 4 ), indicating that this component of the transgene also conferred resistance. However, when inoculated with PVX-CP, all T1 transgenic plants (both CTV1 and CTV2) were susceptible and showed symptoms by 7 days postinoculation (Table 4).

The lack of resistance to PVX-CP was somewhat surprising. Therefore, we next examined for resistance by inoculating CTV1 plants with recombinant PVX carrying chimeric sequences, containing CP and other components of the transgene (PVX-CP-p20 and PVX-CP-UTR). In these tests, CTV1 plants showed good resistance to these PVX constructs (Table 4). These phenotypic observations again were confirmed with RT-PCR analysis, which confirmed resistance or susceptibility.

Resistance is PTGS mediated. In order to determine whether the resistance in the CTV1 and CTV2 transgenic plants was mediated by PTGS, we performed northern hybridization analysis for siRNAs from both transgenic and nontransgenic N. benthamiana plants. Specific $\approx 22$ - to 24 -nt duplex RNA (siRNA) molecules specific to chimeric transgene sequences (CP-p20-UTR for CTV1 and CP-p23-UTR for CTV2) were detected in both uninoculated and resistant transgenic plants, but not in uninoculated nontransgenic plants (Fig. 6), demonstrating that the transgenic plants were silenced for the respective transgenes. Strong and intense siRNA signals also were observed in both PVX-p20- or PVX-p23-inoculated susceptible transgenic (Fig. 6A, lane 5 and Fig. 6B, lane 7) and nontransgenic plants (Fig. 6B, lane 5) when

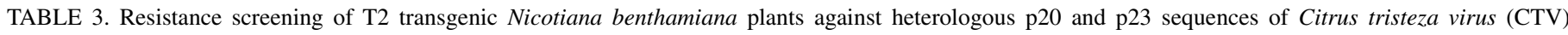
isolates from diverse origin ${ }^{\mathrm{a}}$

\begin{tabular}{|c|c|c|c|c|c|}
\hline \multirow[b]{2}{*}{ Inoculum, CTV isolate ${ }^{b}$} & \multicolumn{2}{|c|}{$\mathrm{T} 2 \mathrm{CTV}^{\mathrm{c}}$} & \multicolumn{2}{|c|}{ T2 CTV2c } & \multirow[b]{2}{*}{ Nontransgenic } \\
\hline & Line $015-1$ & Line $015-2$ & Line $041-1$ & Line 041-2 & \\
\hline \multicolumn{6}{|l|}{ PVX-p20 } \\
\hline CA-65 & $18 / 18$ & $16 / 18$ & $1 / 9$ & $0 / 9$ & $0 / 14$ \\
\hline FL-T30 & $18 / 18$ & $15 / 18$ & $0 / 9$ & $0 / 9$ & $0 / 13$ \\
\hline IND-B227 & $18 / 18$ & $14 / 18$ & $0 / 9$ & $0 / 9$ & $0 / 13$ \\
\hline ISR-VT & $18 / 18$ & $11 / 18$ & $0 / 9$ & $0 / 9$ & $0 / 13$ \\
\hline \multicolumn{6}{|l|}{ PVX-p23 } \\
\hline CA-65 & $0 / 9$ & $0 / 9$ & $18 / 18$ & $18 / 18$ & $0 / 14$ \\
\hline FL-T30 & $0 / 9$ & $0 / 9$ & $17 / 18$ & $16 / 18$ & $0 / 13$ \\
\hline IND-B227 & $0 / 9$ & $0 / 9$ & $18 / 18$ & $14 / 18$ & $0 / 13$ \\
\hline CA-SY568 & $0 / 9$ & $0 / 9$ & $18 / 18$ & $17 / 18$ & $0 / 13$ \\
\hline
\end{tabular}

a Number of nonsymptomatic plants at 7 days postinoculation/total number of inoculated plants.

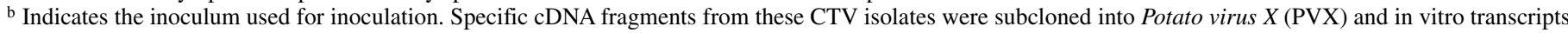
were used for plant inoculation.

${ }^{c}$ Indicates plants used for challenge with given inoculum. 
compared with the resistant transgenic plants (Fig. 6A, lanes 1 through 4 and Fig. 6B, lanes 2 through 4 and 8), indicating that these siRNA signals were most likely due to virus-induced gene silencing (VIGS). Also, because we failed to observe resistance for PVX-CP in our challenge experiments, we probed for siRNAs specific to each component of the chimeric transgene sequences. Northern blot hybridization with CTV-CP-, p20-, and UTR-specific probes showed 22- to 24-nt siRNAs in uninoculated CTV1 plants, but no p20-specific siRNAs in CTV2 plants, as expected. Similarly, with CP-, p23-, and UTR-specific probes, we detected siRNAs in uninoculated CTV2 plants but no p23-specific siRNAs in CTV1 plants (data not shown). Thus, CTV CP-specific siRNAs were present in transgenic plants, even though resistance to PVX$\mathrm{CP}$ was not observed.

\section{DISCUSSION}

In this work, transgenic $N$. benthamiana plants expressing specific CTV sequences were constructed and used to assess resistance to recombinant PVX constructs containing corresponding homologous or heterologous CTV sequences. Even though $N$. benthamiana plants are not CTV hosts, we felt that this approach
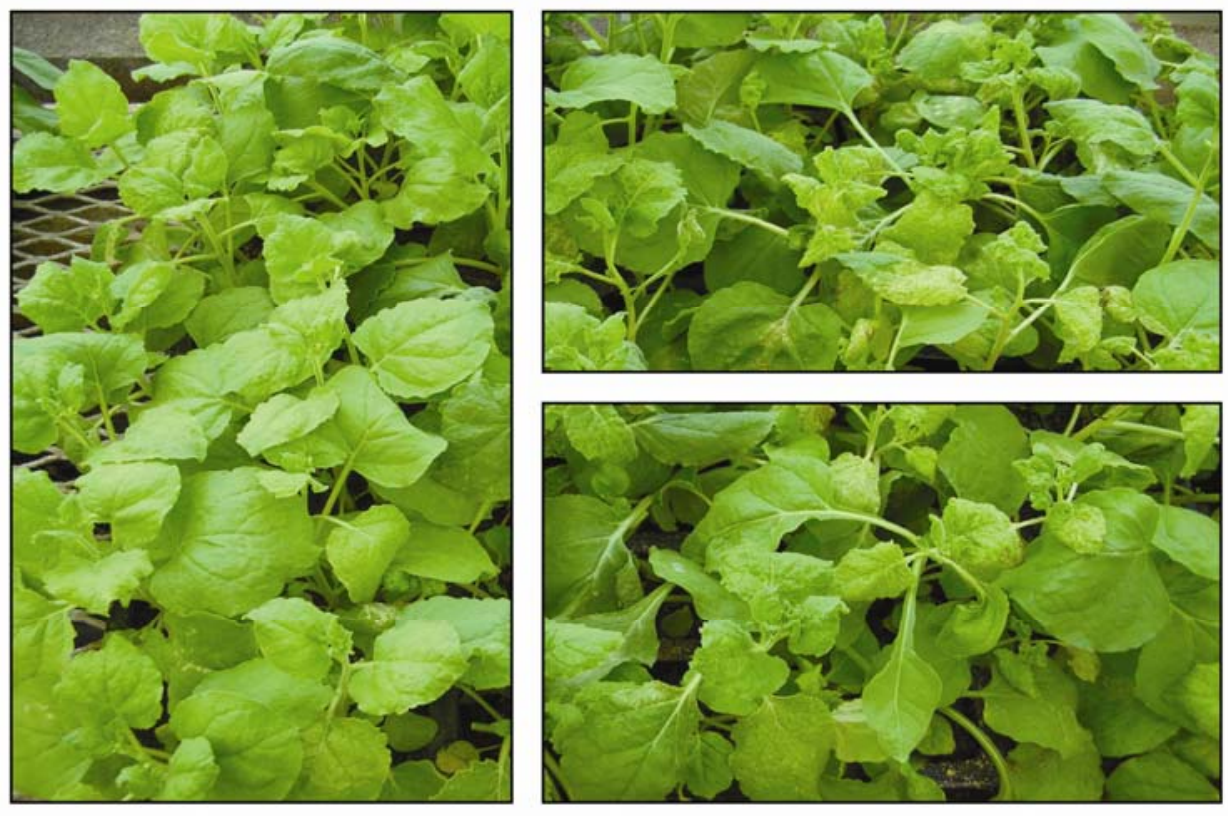

A
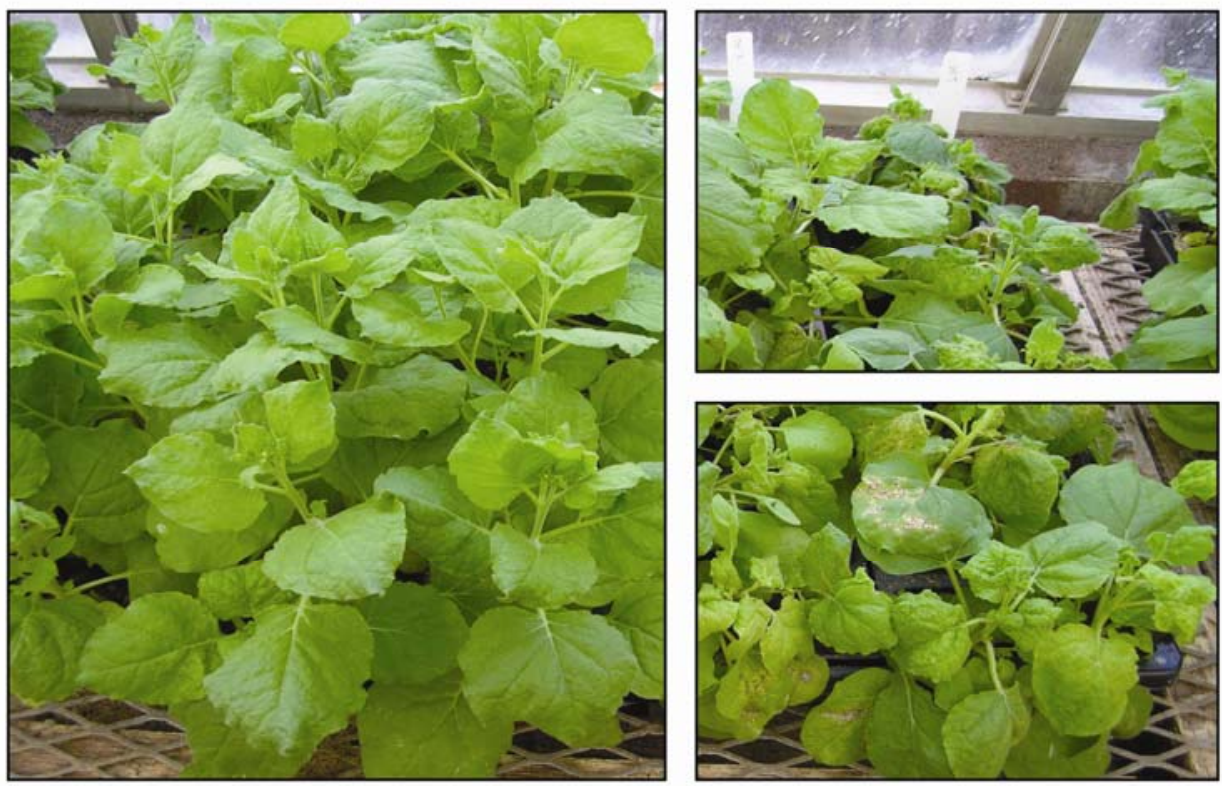

\section{B}

Fig. 4. Transgenic resistance against heterologous Citrus tristeza virus (CTV) sequences. A, Resistance in T2 CTV1 line 015-1 plants against Potato virus $X$ (PVX) containing the p20 sequence from CTV isolate ISR-VT. Transgenic T2 CTV2 line 041-1 (right top) and nontransgenic (right bottom) control plants showed symptoms by 7 days postinoculation (dpi). B, Resistance in T2 CTV2 line 041-1 plants against PVX carrying p23 from isolate CA-SY568. Transgenic T2 CTV1 line 015-1 (right top) and nontransgenic (right bottom) control plants developed necrotic symptoms at 7 dpi. Resistant plants at left showed no symptoms. 
offered benefits for evaluating PTGS-based resistance against CTV. $N$. benthamiana is an herbaceous plant and more amenable to rapid analysis of transgenic progeny than are woody citrus plants and, as shown here, different viruses and transgenes can be evaluated quickly. Our transgenic $N$. benthamiana plants displayed good resistance to the CTV sequences delivered by the PVX vector. Resistance was seen in plants of both the T1 and T2 transgenic lines even when the latter were inoculated with PVX containing p20 (for CTV1) and p23 (for CTV2) sequences from geographically diverse CTV isolates (Table 3), and resistance in $\mathrm{T} 2$ plants was very robust (a high percentage of nonsymptomatic plants).
Inoculating PVX-containing homologous sequences other than p20 (for CTV1) and p23 (for CTV2) revealed two different phenotypes. PVX-UTR-inoculated plants showed resistance, whereas PVX-CP-inoculated plants were susceptible. The reason for the lack of resistance in CTV1 and CTV2 plants when challenged with PVX-CP is not known. In the transgene construct, the $\mathrm{CP}$ sequence is located at the end in the predicted chimeric self-complementary RNA structure; however, CP-specific siRNAs were detected by us in northern blots. It also is interesting to note that there are several reports of developing CTV-CP transgenic citrus plants, but definitive reports of CTV resistance in these plants generally are lacking or somewhat inconclusive $(7,8,12,14,38)$. It

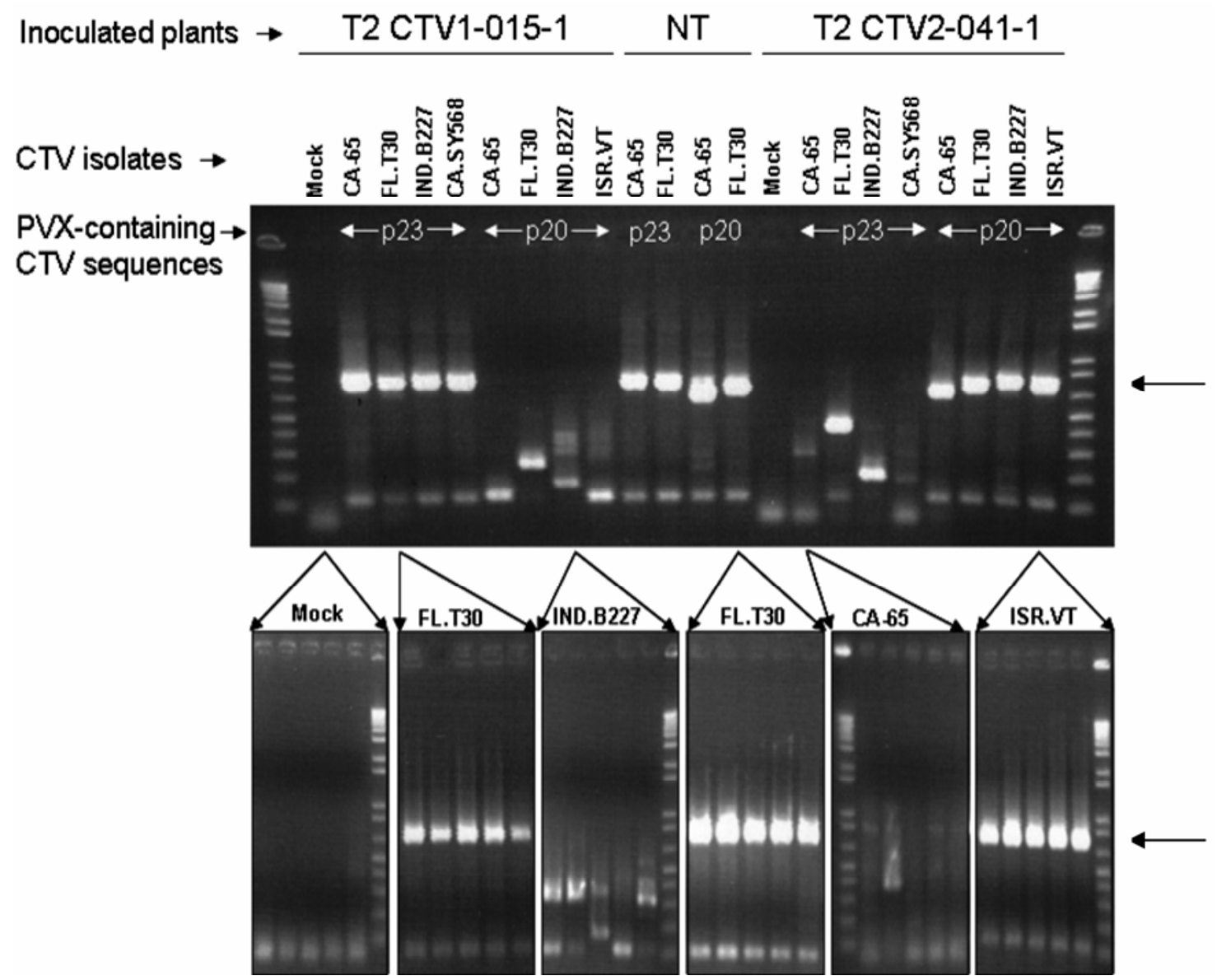

Fig. 5. Reverse-transcriptase polymerase chain reaction analysis showing resistance to heterologous Citrus tristeza virus (CTV) sequences in transgenic plants. T2 CTV1 line 015-1 plants were inoculated with Potato virus X (PVX) carrying p20 sequences of CTV isolates (CA-65, FL.T30, IND.B227, and ISR.VT) and T2 CTV2 line 041-1 plants inoculated with PVX carrying p23 sequences of CTV isolates (CA-65, FL.T30, IND.B227, and CA.SY568). Arrows indicate the expected size product which, if present, indicates susceptibility. Lack of a product indicates resistance. Inoculating nontransgenic and transgenic control plants (T2 CTV1 line 015-1) with each of PVX-p23, and nontransgenic as well as T2 CTV2 line 041-1 plants inoculated with each of PVX-p20 constructs, revealed that these plants were susceptible to nonhomologous recombinant PVX infection. Bottom panel indicates analyses of five individual plants for each of the samples indicated above. Note that p20 product from CA-65 isolate is smaller by 55 nucleotides (nt) than others due to an internal SalI site present in this isolate at the 55-nt position from the $3^{\prime}$ end of the original p23 fragment and was cleaved off during digestion with SalI before cloning into PVX.

TABLE 4. Resistance screening of T1 transgenic Nicotiana benthamiana plants against homologous sequences of Citrus tristeza virus from capsid protein (CP), untranslated region (UTR), CPUTR, CPp20, and p20UTR ${ }^{\mathrm{a}}$

\begin{tabular}{|c|c|c|c|c|c|}
\hline \multirow[b]{2}{*}{ Inoculum $^{\text {b }}$} & \multirow[b]{2}{*}{ Nontransgenic } & \multicolumn{2}{|c|}{ T1 CTV1 ${ }^{\mathrm{c}}$} & \multicolumn{2}{|c|}{$\mathrm{T} 1 \mathrm{CTV}^{\mathrm{c}}$} \\
\hline & & Line 009 & Line 015 & Line 002 & Line 041 \\
\hline PVX-CP & $0 / 18$ & $0 / 18$ & $0 / 18$ & $0 / 18$ & $0 / 18$ \\
\hline PVX-UTR & $0 / 18$ & $10 / 18$ & $14 / 18$ & $17 / 18$ & $15 / 18$ \\
\hline PVX-CPUTR & $0 / 18$ & $7 / 18$ & $11 / 18$ & $15 / 18$ & $12 / 18$ \\
\hline PVX-CPp20 & $0 / 18$ & $8 / 18$ & $13 / 18$ & NT & NT \\
\hline PVX-p20UTR & $0 / 18$ & $12 / 18$ & $17 / 18$ & NT & NT \\
\hline
\end{tabular}

a Number of nonsymptomatic plants at 20 days postinoculation/total number of inoculated plants; NT $=$ not tested.

b $\mathrm{PVX}=$ Potato virus $X$. Figure 2 provides description of constructs used to generate inocula.

c Plants challenged with corresponding inoculum. 


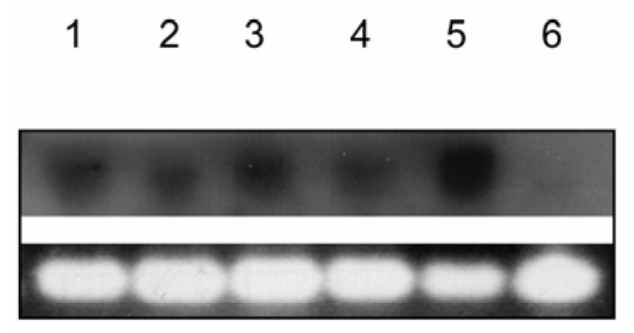

A

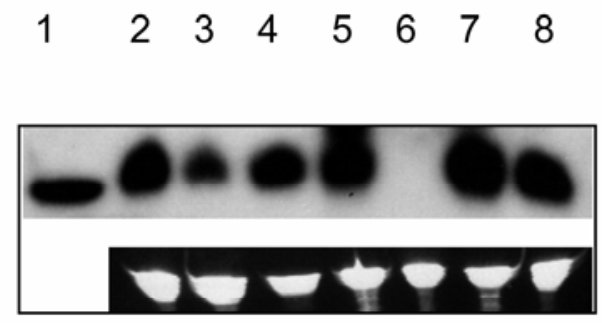

B

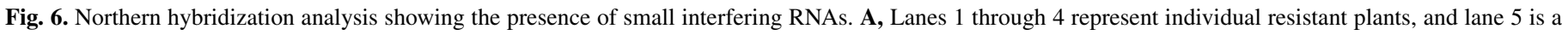

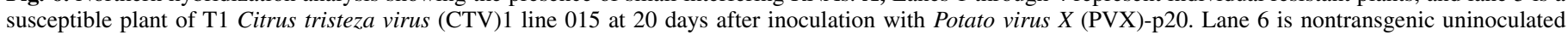

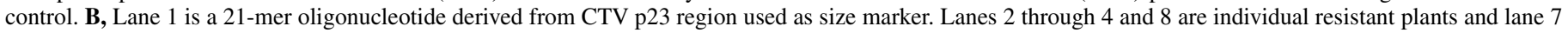

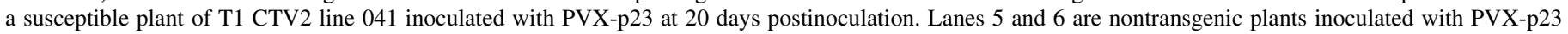
and an uninoculated control, respectively. RNA loading control shows ethidium bromide-stained agarose gel (A, bottom) and polyacrylamide gel (B, bottom).

seems likely that at least one of these reports should have led to robust resistance if the CTV CP was an effective resistance gene. The CP sequence used by us here was only $274 \mathrm{nt}$; however, whether or not the CTV CP alone may be a good transgene for conferring CTV resistance seems questionable. However, when the CP sequence was fused with p20 or UTR and expressed from PVX, our CTV1 plants showed resistance (Fig. 2B; Table 4). Furthermore, we found resistance in both CTV1 and CTV2 plants when challenged with PVX expressing only the UTR sequence (279 nt), which is similar in size to the CP sequence (Fig. 2A; Table 4).

In our resistant $N$. benthamiana plants, we found $\approx 22$ - to 24 -nt siRNAs specific to CTV1 and CTV2 sequences. The amount of siRNA detected in inoculated and uninoculated plants of resistant CTV1 and CTV2 lines was similar, but less than the siRNA detected for corresponding susceptible transgenic (compare Fig. 6A, lane 5 with lanes 1 to 4 for CTV1 and compare Fig. 6B, lane 7 with lanes 2 to 4 and 8 for CTV2) and inoculated nontransgenic (Fig. 6B, lane 5) plants. Thus, the presence of siRNAs in the resistant transgenic plants supports the idea that the resistance was mediated by PTGS and that the siRNAs obtained from susceptible (nontransgenic and transgenic) plants likely were due to VIGS (26). Interestingly, we also detected siRNAs specific to CTV CP sequences in RNA gel blot analysis (data not shown), even though all the CTV1 and CTV2 lines examined here were susceptible to PVX-CP.

Our results suggest that it is worth considering using this strategy for developing CTV resistance in citrus. First, the transgene sequences used here were from highly conserved regions of the CTV genome and thus, hopefully, can target the diversity found among distinct CTV isolates. Second, the chimeric transgene constructs contain sequences from three CTV genomic regions, including CTV-encoded silencing suppressors. Silencing suppressors often are reported to be pathogenicity determinants, at least in N. benthamiana plants (5), and the CTV-encoded p23 is reported to be a symptom determinant in some citrus hosts (11). Although we showed the resistance here to be CTV sequence specific (not to PVX alone) in $N$. benthamiana plants, it remains important to determine whether these or similar genes, or even PTGS, will work effectively so as to give effective control against CTV in citrus plants. There are reports of PTGS-mediated virus resistance in perennial trees (e.g., papaya [34] and stone fruit [18]) and, thus, citrus, as perennial plants, also should be amenable to this strategy. What may be more important, however, is whether PTGS resistance will be effective in the phloem against a phloem-limited virus like CTV. The recombinant PVX resistance seen here was effective and specific, but PVX is largely a mesophyll-infecting plant virus. Infection begins in mesophyll tissues and then moves systemically. In contrast, natural infection by CTV occurs via aphid inoculation to the phloem, and the infection remains in phloem tissues. Also, as noted above, other attempts to generate transgenic CP-mediated CTV resistance in citrus have been unsuccessful (12). However, a recent report suggests that PTGS-mediated resistance against CTV in transgenic Mexican Lime plants has been achieved (10). These plants were transgenic for the CTV-encoded p23. The authors argue that the resistance was RNA-mediated; however, siRNAs were not detected in transgenic, uninoculated resistant plants, and genetically identical siblings were not consistent in their response to CTV infection. Some plants showed resistance and some susceptibility, again suggesting that achieving effective PTGS-mediated resistance against CTV may be somewhat more problematic than it has been for $N$. benthamiana plants. However, effective PTGS-mediated resistance has been developed against some phloem-limited viruses such as Barley yellow dwarf virus (35), suggesting that phloemlimited viruses, per se, can be targeted by PTGS. We have regenerated citrus seedlings transgenic for CTV1 and CTV2 and currently are evaluating their resistance or susceptibility to various CTV isolates.

\section{ACKNOWLEDGMENTS}

This work was supported in part by the California Citrus Research Board, the University of California BioSTAR/UC Discovery grants program, and the United States Department of Agriculture. We thank S. Uratsu for help and for comments on the manuscript, J. Bowman for providing pRNA69, D. Tricoli for help with transgenic plants, and J. Hyle for isolation of CTV RNA from the Beltsville CTV isolate collection.

\section{LITERATURE CITED}

1. Asins, M. J., Bernet, G. P., Ruiz, C., Cambra, M., Guerri, J., and Carbonell, E. A. 2004. QTL analysis of Citrus tristeza virus-citradia interaction. Theor. Appl. Genet. 108:603-611.

2. Bandyopadhaya, R., and Frederiksen, R. A. 1999. Contemporary global movement of emerging plant diseases. Ann. N.Y. Acad. Sci. 894:28-36.

3. Bernet, G. P., Breto, M. P., and Asins, M. J. 2004. Expressed sequence enrichment for candidate gene analysis of citrus tristeza virus resistance. Theor. Appl. Genet. 108:592-602.

4. Bernstein, E., Caudy, A. A., Hammond, S. M., and Hannon, G. J. 2001. Role for a bidentate ribonuclease in the initiation step of RNA interference. Nature 409:363-366.

5. Brigneti, G., Voinnet, O., Li, W. X., Ji, L. H., Ding, S. W., and Baulcombe, D. C. 1998. Viral pathogenicity determinants are suppressors 
of transgene silencing in Nicotiana benthamiana. EMBO J. 17:67396746.

6. Costa, A. S., and Muller, G. W. 1980. Tristeza control by cross protection: A U.S.-Brazil cooperative success. Plant Dis. 64:538-541.

7. Dominguez, A., de Mendoza, A. H., Guerri, J., Cambra, M., Navarro, L., Moreno, P., and Pena, L. 2002. Pathogen-derived resistance to Citrus tristeza virus (CTV) in transgenic Mexican lime (Citrus aurantifolia (Christ.) Swing.) plants expressing its p25 coat protein gene. Mol. Breed. 10:1-10.

8. Dominguez, A., Fagoaga, C., Navarro, L., Moreno, P., and Pena, L. 2002. Regeneration of transgenic citrus plants under non selective conditions results in high-frequency recovery of plants with silenced transgenes. Mol. Genet. Genomics 267:544-556.

9. Elbashir, S. M., Lendeckel, W., and Tuschl, T. 2001. RNA interference is mediated by 21- and 22-nucleotide RNAs. Genes Dev. 15:188-200.

10. Fagoaga, C., Lopez, C., Hermoso de Menndoza, A., Moreno, P., Navarro, L., Flores, R., and Pena, L. 2006. Post-transcriptional gene silencing of the p23 silencing suppressor of Citrus tristeza virus confers resistance to the virus in transgenic Mexican lime. Plant Mol. Biol. 60:153-165.

11. Fagoaga, C., Lopez, C., Moreno, P., Navarro, L., Flores, R., and Pena, L. 2005. Viral-like symptoms induced by the ectopic expression of the p23 gene of Citrus tristeza virus are citrus specific and do not correlate with the pathogenicity of the virus strain. Mol. Plant-Microbe Interact. 18:435445.

12. Febres, V. J., Niblett, C. L., Lee, R. F., and Moore, G. A. 2003. Characterization of grapefruit plants (Citrus paradisi Macf.) transformed with citrus tristeza closterovirus genes. Plant Cell Rep. 21:421-428.

13. Foster, T. M., Lough, T. J., Emerson, S. J., Lee, R. H., Bowman, J. L., Forster, R. L. S., and Lucas, W. J. 2002. A surveillance system regulates selective entry of RNA into the shoot apex. Plant Cell 14:1497-1508.

14. Ghorbel, R., Dominguez, A., Navarro, L., and Pena, L. 2000. High efficiency genetic transformation of sour orange (Citrus aurantium) and production of transgenic trees containing the coat protein gene of Citrus tristeza virus. Tree Physiol. 20:1183-1189.

15. Gutierrez, E. M. A., Luth, D., and Moore, G. A. 1997. Factors affecting Agrobacterium-mediated transformation in Citrus and production of sour orange (Citrus aurantium L.) plants expressing the coat protein gene of Citrus tristeza virus. Plant Cell Rep. 16:745-753.

16. Hamilton, A. J., and Baulcombe, D. C. 1999. A species of small antisense RNA in posttranscriptional gene silencing in plants. Science 286:950-952

17. Hammond, S. M., Bernstein, E., Beach, D., and Hannon, G. J. 2000. An RNA-directed nuclease mediates post-transcriptional gene silencing in Drosophila cells. Nature 404:293-296.

18. Hily, J. M., Scorza, R., Malinowski, T., Zawadzka, B., and Ravelonandro, M. 2004. Stability of gene silencing-based resistance to Plum pox virus in transgenic plum (Prunus domestica L.) under field conditions. Transgenic Res. 13:427-436.

19. Karasev, A. V. 2000. Genetic diversity and evolution of closteroviruses. Annu. Rev. Phytopathol. 38:293-324.

20. Karasev, A. V., Boyko, V. P., Gowda, S., Nikolaeva, O. V., Hilf, M. E., Koonin, E. V., Niblett, C. L., Cline, K., Gumpf, D. J., and Lee, R. F. 1995. Complete sequence of the citrus tristeza virus RNA genome. Virology 208:511-520.

21. Kong, P., Rubio, L., Polek, M., and Falk, B. W. 2000. Population structure and genetic diversity within California Citrus tristeza virus (CTV) isolates. Virus Genes 21:139-145.

22. Lindbo, J. A., and Dougherty, W. G. 1992. Pathogen-derived resistance to a potyvirus: Immune and resistant phenotypes in transgenic tobacco expressing altered forms of a potyvirus coat protein nucleotide sequence. Mol. Plant-Microbe Interact. 5:144-153.

23. Lindbo, J. A., and Dougherty, W. G. 2005. Plant pathology and RNAi: A brief history. Annu. Rev. Phytopathol. 43:191-204.

24. Lindbo, J. A., Silva-Rosales, L., Proebsting, W. M., and Dougherty, W. G.1993. Induction of a highly specific antiviral state in transgenic plants: Implications for regulation of gene expression and virus resistance. Plant Cell 5:1749-1759.

25. Lu, R., Folimonov, A., Shintaku, M., Li, W. X., Falk, B. W., Dawson, W. O., and Ding, S.W. 2004. Three distinct suppressors of RNA silencing encoded by a $20-\mathrm{kb}$ viral RNA genome. Proc. Natl. Acad. Sci. USA 101:15742-15747.

26. Macdiarmid, R. 2005. RNA Silencing in productive virus infections. Annu. Rev. Phytopathol. 43:523-544.

27. McBride, K. E., and Summerfelt, K. R. 1990. Improved binary vectors for Agrobacterium-mediated plant transformation. Plant Mol. Biol. 14:269-276.

28. Molnar, A., Csorba, T., Lakatos, L., Varallyay, E., Lacomme, C., and Burgyan, J. 2005. Plant virus-derived small interfering RNAs originate predominantly from highly structured single-stranded viral RNAs. J. Virol. 79:7812-7818.

29. Napoli, C., Lemieux, C., and Jorgensen, R. 1990. Introduction of a chimeric chalcone synthase gene into petunia results in reversible cosuppression of homologous genes in trans. Plant Cell 2:279-289.

30. Nykanen, A., Haley, B., and Zamore, P. D. 2001. ATP requirements and small interfering RNA structure in the RNA interference pathway. Cell 107:309-321.

31. Roistachar, C. N., and Moreno, P. 1991. The worldwide threat from destructive isolates of citrus tristeza virus-a review. Pages 100-107 in: Proc. 11 Conf. Int. Org. Citrus Virol.

32. Rubio, L., Ayllon, M. A., Kong, P., Fernandez, A., Polek, M., Guerri, J., Moreno, P., and Falk, B. W. 2001. Genetic variation of Citrus tristeza virus isolates from California and Spain: Evidence for mixed infections and recombination. J. Virol. 75:8054-8062.

33. Smith, N. A., Singh, S. P., Wang, M. B., Stoutjesdijk, P. A., Green, A. G., and Waterhouse, P. M. 2000.Total silencing by intron-spliced hairpin RNAs. Nature 407:319-320.

34. Tennant, P., Fermin, G., Fitch, M. M., Manshardt, R. M., Slightom, J. L., and Gonsalves, D. 2001. Papaya ringspot virus resistance of transgenic Rainbow and SunUp is affected by gene dosage, plant development, and coat protein homology. Eur. J. Plant Pathol. 107:645-653.

35. Wang, M. B., Abbott, A. C., and Waterhouse, P. M. 2000. A single copy of a virus-derived transgene encoding hairpin RNA gives immunity to barley yellow dwarf virus. Mol. Plant Pathol. 1:347-356.

36. Wang, M. B., and Waterhouse, P. M. 2000. High-efficiency silencing of a beta-glucuronidase gene in rice is correlated with repetitive transgene structure but is independent of DNA methylation. Plant Mol. Biol. 43:67-82.

37. Wesley, S. V., Helliwell, C. A., Smith, N. A., Wang, M. B., Rouse, D. T., Liu, Q., Gooding, P. S., Singh, S. P., Abbott, D., Stoutjesdijk, P. A., Robinson, S. P., Gleave, A. P., Green, A. G., and Waterhouse, P. M. 2001. Construct design for efficient, effective and high-throughput gene silencing in plants. Plant J. 27:581-590.

38. Yang, Z. N., Ingelbrecht, I. L., Louzada, E., Skaria, M., and Mirkov, T. E. 2000. Agrobacterium-mediated transformation of the commercially important grapefruit cultivar Rio Red (Citrus paradisi Macf.). Plant Cell Rep. 19:1203-1211.

39. Zamore, P. D. 2001. RNA interference: Listening to the sound of silence. Nat. Struct. Biol. 8:746-750. 